\title{
Peroxisome proliferator-activated receptor-y stimulates the synthesis of monounsaturated fatty acids in dairy goat mammary epithelial cells via the control of stearoyl-coenzyme A desaturase
}

\author{
H. B. Shi, ${ }^{,}$J. Luo, ${ }^{* 1}$ D. W. Yao, ${ }^{*}$ J. J. Zhu, ${ }^{*}$ H. F. Xu, ${ }^{*}$ H. P. Shi, ${ }^{*}$ and J. J. Loor† \\ *Shaanxi Key Laboratory of Molecular Biology for Agriculture, College of Animal Science and Technology, Northwest A\&F University, Yangling, \\ Shaanxi, 712100, P.R. China \\ †Mammalian NutriPhysioGenomics, Department of Animal Sciences and Division of Nutritional Sciences, University of Illinois, Urbana 61801
}

\begin{abstract}
In rodents, peroxisome proliferator-activated receptor- $\gamma$ (PPARG) plays a crucial role in fatty acid (FA) metabolism through regulation of gene expression, including stearoyl-coenzyme A desaturase (SCD), which is the rate-limiting enzyme for the biosynthesis of monounsaturated FA. However, whether or how PPARG regulates the activity of mammary SCD in ruminants is unknown. This study explored the potential role of PPARG isoforms in regulating $S C D$ mRNA expression in lactating goat mammary epithelial cells (GMEC). Using quantitative real-time PCR, we observed a positive correlation between PPARG and $S C D$ expression in the goat mammary gland at peak lactation. Overexpression of both PPARG1 and PPARG2 in GMEC increased markedly the expression of $S C D$, the concentration of $16: 1$ and $18: 1$, and the desaturation indices of 16:1 and 18:1. The PPARG ligand rosiglitazone further increased $S C D$ expression and desaturation indices in GMEC, overexpressing PPARG1 and PPARG2. Incubation with rosiglitazone alone increased the expression of $S C D$, but did not alter the concentration of 16- to 18-carbon FA or their desaturation indices. The results provide evidence that PPARG regulates the expression and activity of SCD in GMEC. As such, PPARG may contribute to regulation of SCD and monounsaturated FA synthesis during lactation.
\end{abstract}

Key words: lactation, nutrition, metabolism, desaturation

\section{INTRODUCTION}

Ruminant milk is a rich source of MUFA, which have been recognized to be beneficial for the nutrition of humans (Haenlein, 2004; Pieniak-Lendzion and Niedziółka, 2004). In mammalian lipogenic cells, the

Received June 6, 2013.

Accepted August 13, 2013.

${ }^{1}$ Corresponding author: luojun@nwsuaf.edu.cn long-chain FA (LCFA) palmitoleate (16:1) and oleate (18:1) can be synthesized endogenously from palmitate (16:0) and stearate (18:0) via the action of the endoplasmic reticulum-bound enzyme stearoyl-CoA desaturase (SCD) (Miyazaki and Ntambi, 2003). In ruminants, polymorphisms of $S C D$ have been associated with a significant proportion of the variation in milk FA composition (Mele et al., 2007; Moioli et al., 2007; Kgwatalala et al., 2009). It has been suggested that $S C D$ is a primary candidate gene for altering the proportion of SFA versus MUFA in milk (Conte et al., 2010). Consistent with its function as a desaturase, strong associations between SCD activity and MUFA in bovine milk have been reported (Kgwatalala et al., 2009; Mele et al., 2009; Jacobs et al., 2013). Furthermore, a link between SCD and the concentration of MUFA was also reported in goats (Shi et al., 2012) and sheep (Reh et al., 2004).

In rodents, the mRNA expression and activity of SCD is sensitive to nutrition, hormonal changes, and also peroxisome proliferators (Ntambi, 1995). In addition, the expression of $S C D$ can be regulated directly by sterol regulatory element-binding factor 1 (SREBF1) in the liver (Horton et al., 2002) or indirectly in adipose via activation of peroxisome proliferator-activated receptor- $\gamma$ (PPARG) by LCFA and subsequent upregulation of SREBF1 (Walkey and Spiegelman, 2008). In ruminants, the fat composition of milk can be regulated by dietary LCFA, at least in part, through their effects on SCD (Peterson et al., 2004; Bernard et al., 2005).

In vitro work has provided evidence that several LCFA are natural ligands of PPARG (Kadegowda et al., 2009; Bionaz et al., 2013). Activation of PPARG in bovine mammary epithelial cells (MacT) in response to the chemical ligand rosiglitazone (ROSI) or 16:0 and 18:0 increased the expression of $S C D$ (Kadegowda et al., 2009). Furthermore, ROSI increased the desaturation index of 16:1 in rat (Way et al., 2001) and human adipose tissue (Risérus et al., 2005). To date, 2 protein isoforms of PPARG (PPARG1 and PPARG2) have been discovered (Elbrecht et al., 1996). The 2 isoforms 
are a result of alternative promoter usage and alternative splicing at the $5^{\prime}$ end of the gene; PPARG1 lacks 30 additional amino acids at the $\mathrm{N}$ terminus compared with PPARG2 (Rosen and Spiegelman, 2001).

Compared with rodents and humans, much less is known about the link between PPARG activation and the mRNA expression of SCD in the lactating ruminant mammary gland, and if such an effect leads to changes in the LCFA composition. Our previous work with goat mammary epithelial cells (GMEC) demonstrated upregulation of $S C D$ expression after treatment with ROSI and downregulation of $S C D$ after the knockdown of PPARG (Shi et al., 2013). Based on the data with ruminant mammary cells, we hypothesized that PPARG can regulate the mRNA expression and activity of SCD in MEC. To test this hypothesis, we overexpressed the 2 known PPARG isoforms in GMEC, stimulated the GMEC with ROSI, and studied the effects on the expression of $S C D$ and the composition of LCFA.

\section{MATERIALS AND METHODS}

\section{Goat Mammary Gland Tissue Collection}

The Animal Care and Use Committee of the Northwest A\&F University (Yangling, Shaanxi, China) approved all procedures and experiments. Three-year-old Xinong Saanen dairy goats from the experimental farm at the Northwest A\&F University were used in the study. Mammary gland tissue from goats at peak lactation $(\mathrm{n}=11)$ or the dry period $(\mathrm{n}=3)$ was obtained from the right or left mammary gland via biopsy under sterile conditions. Tissue samples were immediately frozen in liquid nitrogen until RNA extraction.

\section{Cell Culture and Treatments}

The GMEC were isolated from peak lactation Xinong Saanen goats as described previously (Wang et al., 2010). Briefly, cells were incubated at $37^{\circ} \mathrm{C}$ in $5 \%$ $\mathrm{CO}_{2}$ and air. Culture medium was changed every 24 $\mathrm{h}$. The medium was composed of Dulbecco's modified eagle medium/nutrient mixture F-12 (DMEM/F12; HyClone Laboratories, Beijing, China) containing insulin (5 mg/L; Sigma, St. Louis, MO), hydrocortisone (5 mg/L; Sigma), penicillin/streptomycin (10k unit/L; Harbin Pharmaceutical Group Co. Ltd., Harbin, China), epidermal growth factor (1 mg/L; Sigma), and fetal bovine serum (10\%, Gibco Products International Inc., Langley, OK). The details of the cell culture were recently described by our group (Lin et al., 2013; Shi et al., 2013). We used $50 \mu \mathrm{mol} / \mathrm{L}$ of ROSI [dissolved in dimethyl sulfoxide (DMSO); Sigma] for pharmacological treatment of GMEC at 80 to $90 \%$ confluence. Cells were harvested at $0,6,12$, and $24 \mathrm{~h}$ for total RNA extraction or LCFA analysis.

\section{Adenovirus Generation and Overexpression of PPARG1 and PPARG2}

The cDNA of dairy goat PPARG1 (GenBank ID no. HQ589347.1; http://www.ncbi.nlm.nih.gov/genbank/) was cloned as previously described (Shi et al., 2013). The PPARG2 cDNA (GenBank ID no. JN854246) was cloned from GMEC using the following primers: sense 5'-ATGGGTGAAACCCTGGGAGATGCT-3' and antisense 5'-CTAATACAAGTCCTTGTAGATTTCCTGTAG-3'. The coding sequences of PPARG1 and PPARG2 were subcloned into the pAdTrack-CMV plasmid vector between the XhoI and ScalI (New England BioLabs Inc., Ipswich, MA) restriction sites to generate pAdTrack-CMV-PPARG1 and pAdTrack-CMVPPARG2 vectors. These 2 vectors were inserted into an adenoviral vector (pAdEasy-1) to generate adenoviral plasmids in BJ5183 cells. The adenoviral plasmids linearized by PacI (New England BioLabs Inc.) were transfected into $293 \mathrm{~A}$ cells to generate the adenoviruses pAd-PPARG1 and pAd-PPARG2. The whole process for adenovirus generation and proliferation was carried out as previously described (Ostapchuk and Hearing, 2005; Gou et al., 2007; Luo et al., 2007).

The transfected 293A cells were cultured in the basal DMEM medium (Gibco Products International Inc.) containing $10 \%$ fetal bovine serum. The GMEC at about $80 \%$ confluence were transfected with adenovirus supernatant. The adenovirus containing green fluorescent protein (Ad-GFP) was used as a negative control. Transfected GMEC were cultured with ROSI or DMSO after $24 \mathrm{~h}$ of the initial culture and then harvested at $48 \mathrm{~h}$ (24 h later).

\section{Total RNA Extraction, Purification, and Real-Time Quantitative PCR}

Total RNA from goat mammary gland tissue was extracted with TRIzol reagent (Invitrogen Corp., Carlsbad, CA) and the RNAprep pure cell kit (Tiangen Biotech Co. Ltd., Beijing, China) according to the manufacturer instructions. The RNA was treated with DNase (Tiangen Biotech Co. Ltd.) to remove genomic DNA contamination. The cDNA was synthesized from $0.5 \mu \mathrm{g}$ of purified total RNA using the PrimeScript RT kit (Takara Bio Inc., Otsu, Japan) according to the manufacturer instructions. Real-time quantitative PCR (qPCR) was performed according to the manufacturer instructions using SYBR green (SYBR Premix Ex Taq II, Perfect Real Time; Takara Bio Inc.). The sequences for the primers of PPARG, SREBF1, SCD, FA synthase 
$(\boldsymbol{F A S N})$, and acetyl-CoA carboxylase $\alpha(\boldsymbol{A C A} \boldsymbol{C A})$ used in the study were reported previously (Shi et al., 2013). All the qPCR reactions were performed in a Bio-Rad CFX96 sequence detector (Bio-Rad Laboratories Inc., Hercules, CA) and data were normalized to GAPDH (Shi et al., 2013). Every sample was analyzed in triplicate.

\section{FA Analysis}

Total lipid was extracted from approximately $100 \mathrm{mg}$ of GMEC using $2 \mathrm{~mL}$ of chloroform/methanol (Sigma; $3: 1$, vol/vol) as previously described (Wang et al., 2012). Methylated lipid samples were analyzed by gas chromatography (Agilent 7890A; Agilent Technologies Inc., Santa Clara, CA) using an HP-5 column (Agilent Technologies Inc.). The relative proportions of FA were determined as percentages of the total peak areas that could be identified (Wang et al., 2012).

\section{Statistical Analysis}

Each treatment was replicated 3 times and results are expressed as mean \pm standard error of the mean. Data of qPCR were analyzed relative to the control using the $2^{-\Delta \Delta \mathrm{Ct}}$ method, where $\mathrm{Ct}$ is the cycle threshold. The data for each FA was analyzed as a proportion of the total FA. The desaturation index was calculated as the ratio between the desaturation product and the sum of the product and the substrate. For example, a desaturation index of $16: 1=16: 1 /(16: 1+16: 0)$ and a desaturation index of 18:1 = 18:1/(18:1 + 18:0) (Loor and Herbein, 2003). The statistical significance for RNA expression and FA composition was determined by ANOVA using SPSS 19.0 software (SPSS Inc., Chicago, IL). Statistical differences were declared at $P<0.05$.

\section{RESULTS}

\section{PPARG and SCD Expression in Dairy Goat Mammary Gland Tissue}

Relative to the dry period, the expression of PPARG and $S C D$ mRNA increased significantly $(\sim 2$ fold and $\sim 60$ fold, respectively) at peak lactation (Figure 1A). Furthermore, we observed a positive correlation between $P P A R G$ and $S C D$ expression $[\mathrm{r}=0.75 ; P(2$-tailed $)=$ 0.0079 ; Figure 1B].

\section{Activation of PPARG by ROSI Enhances mRNA Expression of SCD}

Despite the lack of a clear increase in expression of $P P A R G$ with ROSI (Figure 2A), the expression of $S C D$ a)

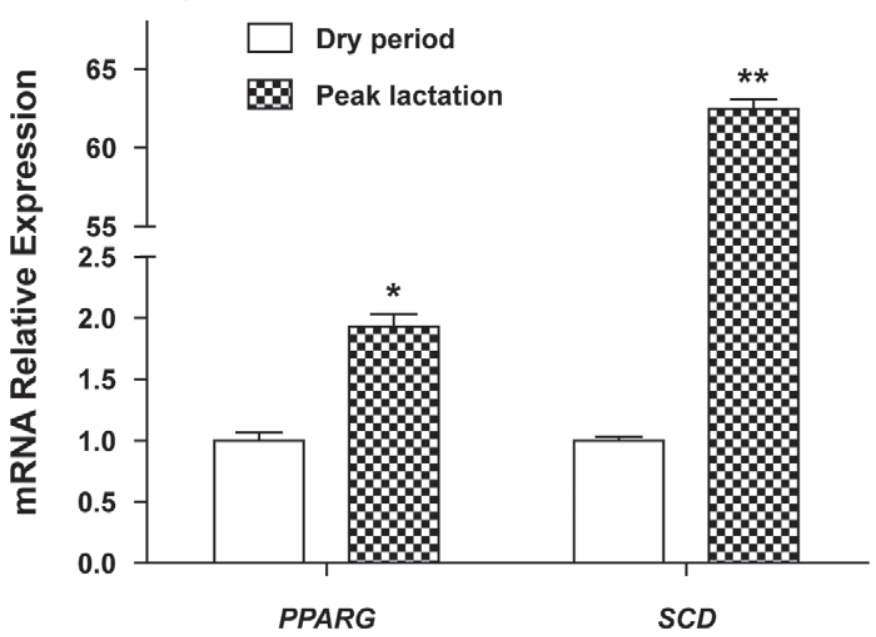

b)

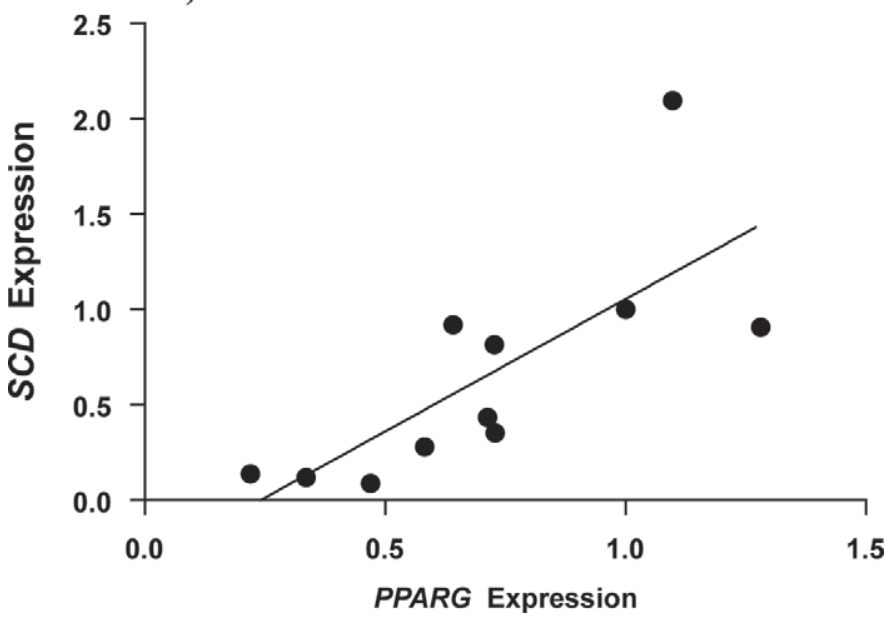

Figure 1. Peroxisome proliferator-activated receptor- $\gamma(P P A R G)$ and stearoyl-CoA desaturase $(S C D)$ expression in dairy goat mammary gland tissue are correlated. Goat mammary gland tissue was collected during the dry period $(\mathrm{n}=3)$ and peak lactation $(\mathrm{n}=11)$ for quantitative PCR. (a) Compared with the dry period, mRNA expression of $P P A R G$ at peak lactation increased significantly $\left({ }^{*} P<0.05\right)$ and that of $S C D$ increased at peak lactation more than 60 fold $(* * P<$ 0.01 ). (b) Relationship between $P P A R G$ and $S C D$ expression in mammary tissue at peak lactation $[\mathrm{n}=11 ; \mathrm{r}=0.75 ; P(2$ tailed $)=0.0079]$

increased significantly at 6,12 , and $24 \mathrm{~h}$ of culture compared with $0 \mathrm{~h}$. Additionally, expression of FASN increased significantly at $24 \mathrm{~h}$, whereas $A C A C A$ and SREBF1 increased at both 12 and $24 \mathrm{~h}$ compared with $0 \mathrm{~h}$.

\section{Effect of ROSI on Concentration of MUFA}

Compared with treatment with DMSO, ROSI had no significant effect on the concentration of 16- to 18-carbon saturated LCFA and MUFA (Figure 2B), or on the desaturation indices of $16: 1$ and 18:1 (Figure 2C and D). 
a)

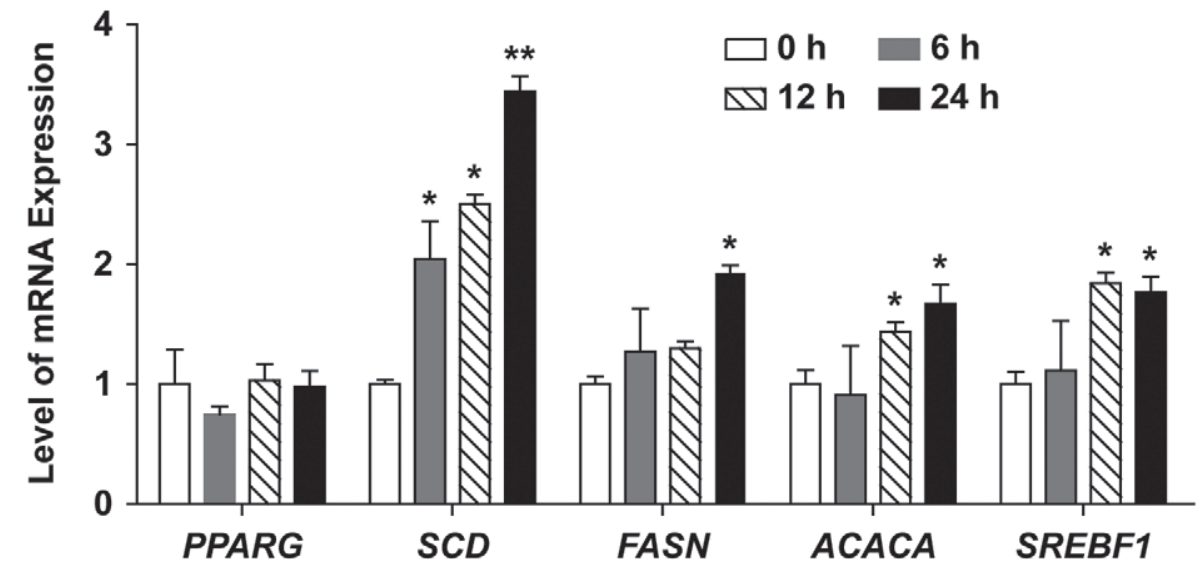

b)

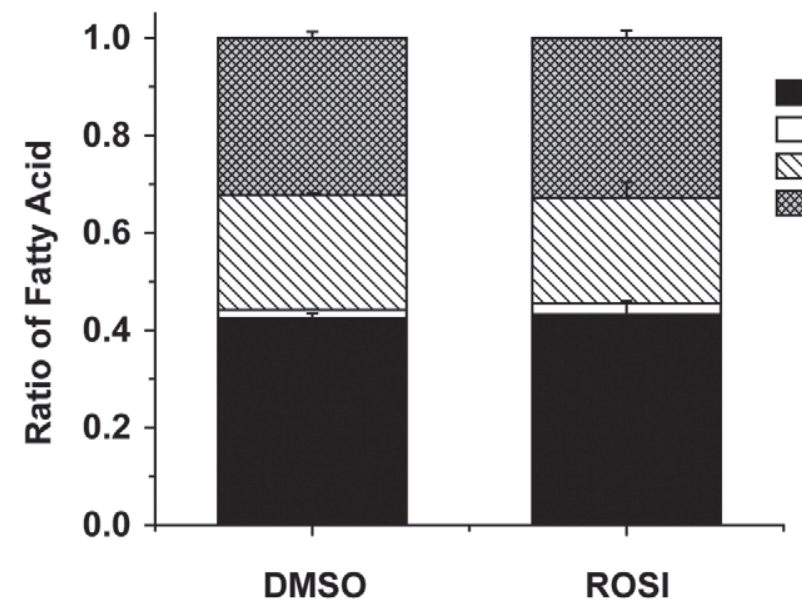

d)

c)
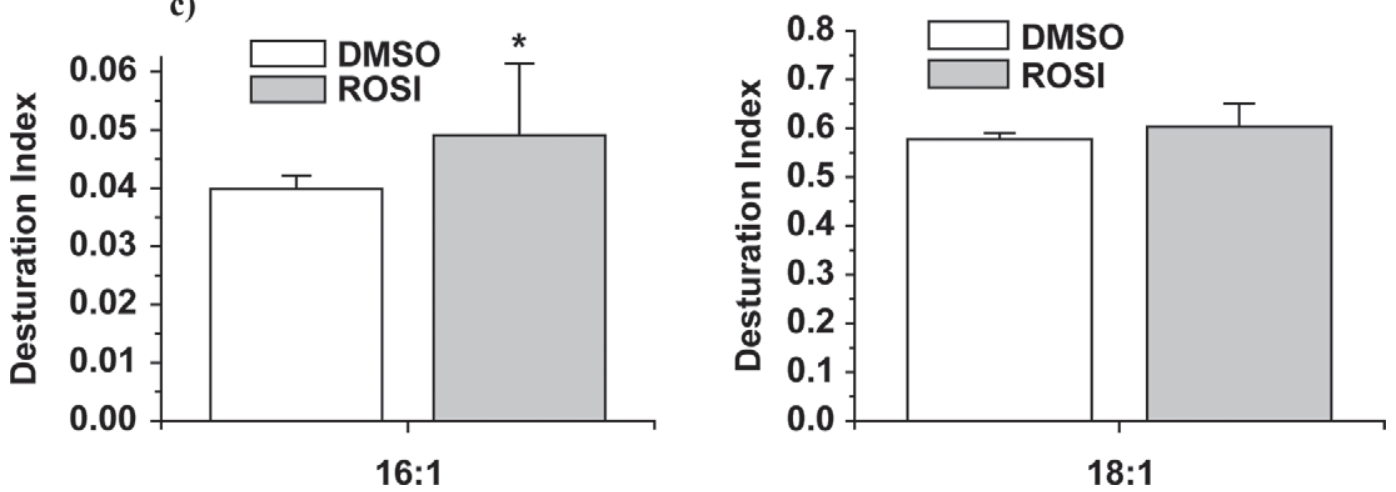

Figure 2. Effect of the pharmaceutical ligand rosiglitazone (ROSI) on gene expression and 16- to 18-carbon FA concentration. Goat mammary epithelial cells (GMEC) were incubated with ROSI and harvested at 0,6, 12, and $24 \mathrm{~h}$ of culture for quantitative PCR. The GMEC were cultured with ROSI or dimethyl sulfoxide (DMSO) and harvested $24 \mathrm{~h}$ later for FA analysis. (a) Level of peroxisome proliferator-activated receptor- $\gamma(P P A R G)$, stearoyl-CoA desaturase $(S C D)$, FA synthase $(F A S N)$, acetyl-CoA carboxylase $\alpha(A C A C A)$, and sterol regulatory elementbinding factor 1 (SREBF1) expression. Data were normalized to $G A P D H$ and expressed relative to 0 h. $* P<0.05$ and $* * P<0.01$ compared with 0 h. (b) Concentration of 16- to 18-carbon FA in cells. Data are reported as ratios of total FA. (c) Desaturation index of 16:1. (d) Desaturation index of 18:1. Values are means \pm SEM for 3 individual experiments. 


\section{Overexpression of PPARG1 Increases the Expression of SCD}

After infection with Ad-PPARG1, the expression of PPARG1 and $S C D$ increased significantly compared with the control group (Ad-GFP+DMSO; Figure 3A and B). An additive effect was also observed where $S C D$ expression increased significantly after treatment with ROSI in the PPARG1 overexpression group. Additionally, the expression of FASN, ACACA, and SREBF1 increased significantly with overexpression of PPARG1 with or without ROSI, but the upregulation was far less than that of $S C D$ (Figure 3B).

\section{Overexpression of PPARG1 Increases Concentration of 16:1 and 18:1}

As shown in Figure 3C, treatment with ROSI had no effect (Ad-GFP+ROSI group vs. Ad-GFP+DMSO group) on the concentration of 16- to 18-carbon FA. However, the ratio of both 16:1 and 18:1 after infection with Ad-PPARG1 adenovirus increased significantly at the expense of 16:0 and 18:0. Furthermore, the desaturation indices of both 16:1 and 18:1 increased significantly after overexpression of PPARG1. The desaturation indices were further enhanced by treatment with ROSI (Figure 3D and E).

\section{Overexpression of PPARG2 Increases the Expression of SCD}

After infection with Ad-PPARG2, the expression of $P P A R G$ and $S C D$ increased significantly compared with the control (Ad-GFP+DMSO; Figure 4A and B). Similar to PPARG1, an additive effect on $S C D$ expression was observed with the ROSI treatment (Figure 4B). In this case, FASN, $A C A C A$, and $S R E B F 1$ were only increased after treatment with ROSI (Figure $4 \mathrm{~B}$ ), and overexpression of PPARG2 suppressed their expression (Figure 4B) compared with PPARG1 overexpression.

\section{Overexpression of PPARG2 Increases Concentration of $16: 1$ and 18:1}

The concentration of both $16: 1$ and 18:1 after infection with Ad-PPARG2 increased significantly but that of 16:0 and 18:0 decreased, suggesting a potent activation of SCD. The desaturation indices of 16:1 (Figure 4D) and 18:1 (Figure 4E) were markedly increased after overexpression of PPARG2 and this increase was enhanced further by ROSI.

\section{DISCUSSION}

Activation of PPARG by ROSI was previously demonstrated to increase the expression of genes related to de novo FA synthesis and $S C D$ in MacT cells (Kadegowda et al., 2009) and GMEC (Shi et al., 2013). The present study is novel in that the regulation of SCD and its intracellular end-product LCFA in GMEC were studied using a combination of activation by ROSI and overexpression of PPARG. The findings from the present study underscore an important role for PPARG in regulating the transcription of $S C D$ and, hence, the composition of MUFA.

The marked increase in expression of both PPARG and $S C D$ in lactating mammary tissue compared with nonlactating tissue along with the strong correlation between PPARG and $S C D$ at peak lactation is in agreement with data from dairy cattle (Bionaz and Loor, 2008). Taken together, our results support the hypothesis that PPARG can regulate the expression of $S C D$ in vivo.

A recent review of the literature led to the conclusion that PPARG plays an important role in ruminant mammary gland lipid metabolism during lactation (Bionaz et al., 2013). Our previous study with GMEC demonstrated that ROSI could enhance the binding of PPARG to the PPAR-response element site (Shi et al., 2013). The marked increase in expression of $S C D$ with ROSI in the present study is in agreement with previous studies in rats (Singh Ahuja et al., 2001), humans (Risérus et al., 2005; Kolak et al., 2007), and bovines (Kadegowda et al., 2009).

The lack of change in the desaturation indices of 16:1 and 18:1 with ROSI agree in part with a previous study in humans, where ROSI increased the desaturation index in adipose tissue of 16:1 but not that of 18:1 (Risérus et al., 2005). The inconsistency may be due to inherent differences in sensitivity to ROSI in adipose tissue versus mammary epithelial cells and (or) inherent differences in sensitivity between ruminants and monogastrics. Differences in insulin sensitivity also may play a role because this hormone is important in the process of mammary lipogenesis in monogastrics but not in ruminants (Bauman et al., 1973).

In the rodent liver, SREBF1 is a key transcription regulator of enzymes in the pathways of cholesterol and FA synthesis and metabolism (Horton et al., 2002). It contributes to the coordinated regulation of pathway components by directly binding to promoters of target genes, including FASN, ACACA, and $S C D$ (Shimano, 2001). Our results demonstrating that activation of PPARG by ROSI upregulated the expression of SREBF1, ACACA, and FASN agree with the results observed in MacT cells (Kadegowda et al., 2009). However, the upregulation of $S C D$ induced by overexpression of PPARG1 was far greater than that of SREBF1, $A C A C A$, and FASN.

The downregulation of $A C A C A$ and SREBF1 with overexpression of PPARG2 was unexpected and sug- 
a)

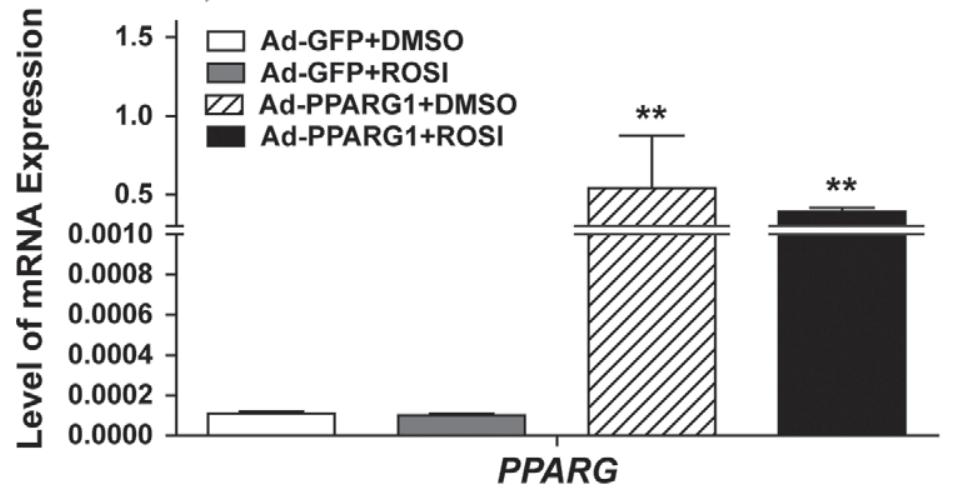

b)

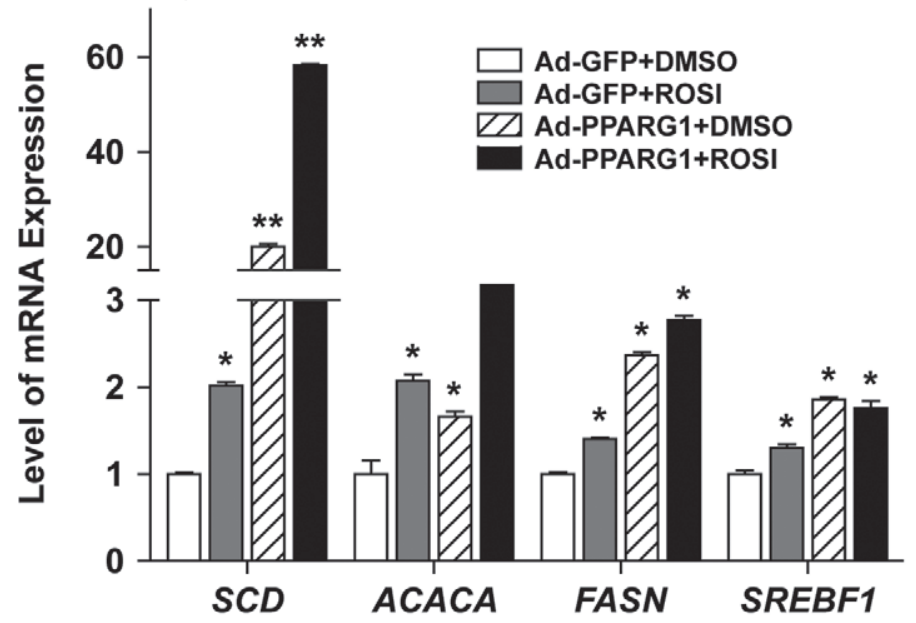

c)

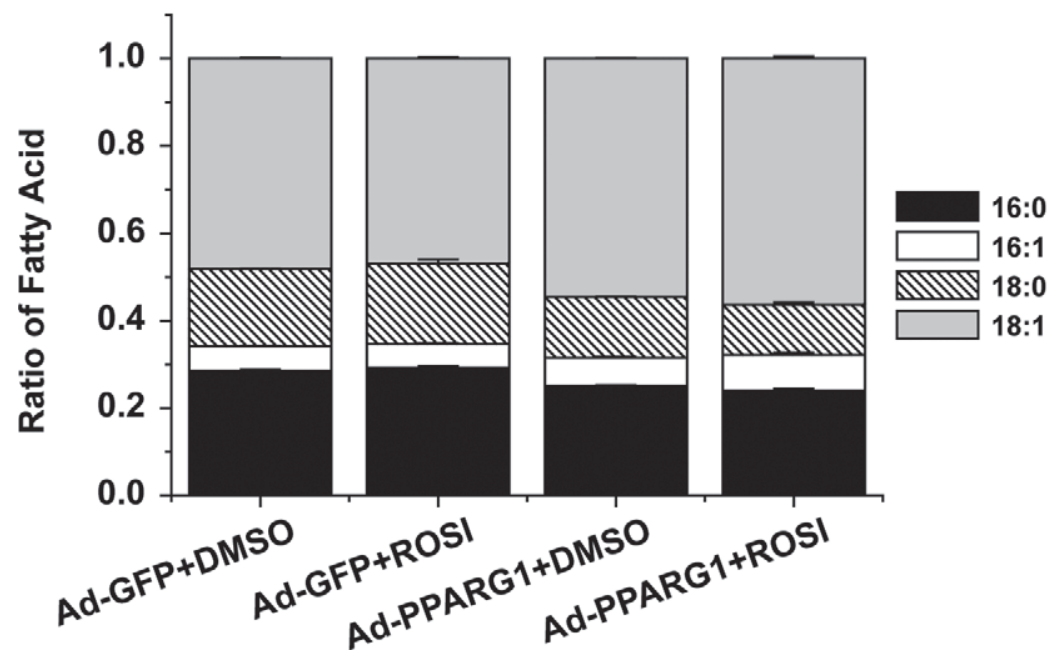

d)

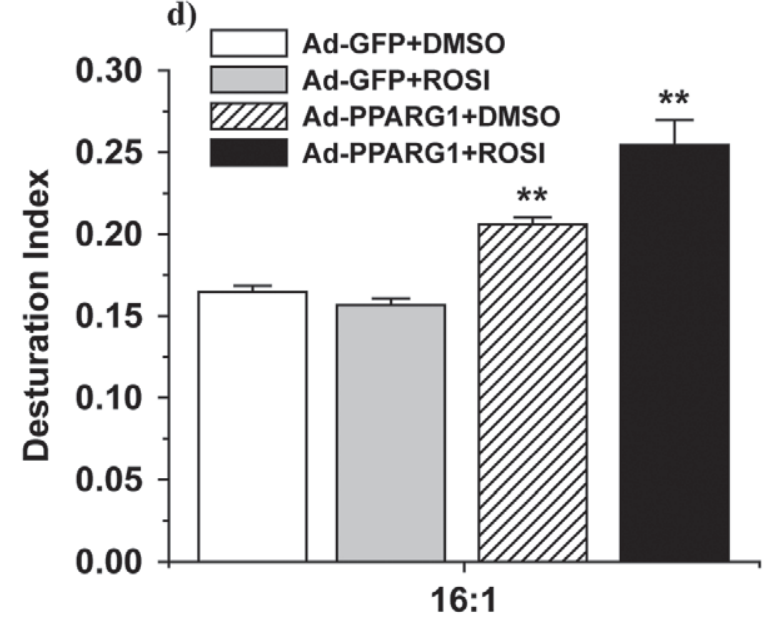

e)

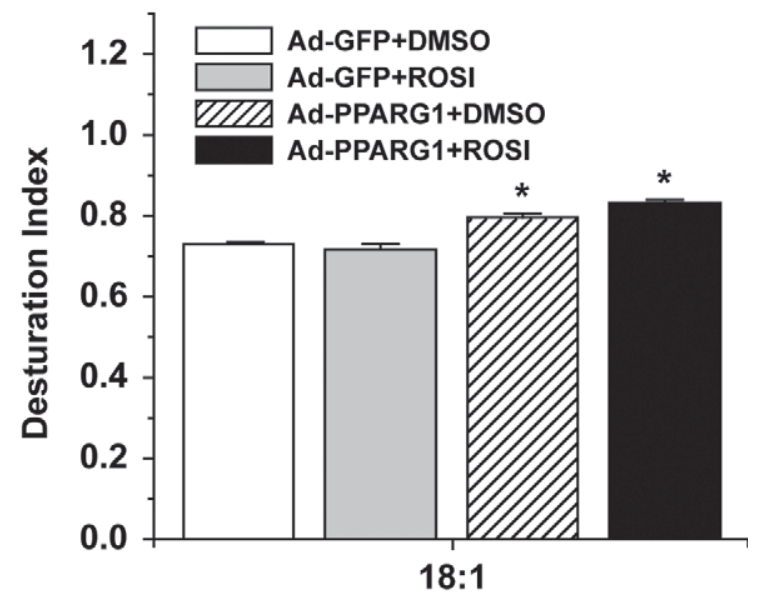

Figure 3. Effect of overexpression of peroxisome proliferator-activated receptor- $\gamma 1$ (PPARG1) on gene expression and FA concentration. After being infected with adenovirus Ad-PPARG1 or adenovirus containing green fluorescent protein (Ad-GFP) for $24 \mathrm{~h}$, the goat mammary epithelial cells (GMEC) were cultured with rosiglitazone (ROSI) or dimethyl sulfoxide (DMSO) for $24 \mathrm{~h}$ and harvested for RNA and lipid extraction. (a) Expression of PPARG after adenovirus-mediated overexpression of PPARG1. (b) Expression of FA synthase (FASN), acetylCoA carboxylase $\alpha(A C A C A)$, stearoyl-CoA desaturase $(S C D)$, and sterol regulatory element-binding factor $1(S R E B F 1)$ after overexpression of PPARG1. (c) Concentration of 16- to 18-carbon FA in cells. Data are reported as ratios of total FA. (d) Desaturation index of 16:1. (e) Desaturation index of 18:1. Values are means \pm SEM for 3 individual experiments. Quantitative PCR data were normalized to GAPDH and expressed relative to the Ad-GFP+DMSO (control) treatment. Data of desaturation indices are relative to the Ad-GFP $+\mathrm{DMSO}$ treatment. ${ }^{*} P$ $<0.05$ and ${ }^{* *} P<0.01$ compared with Ad-GFP+DMSO group cells. 
a)

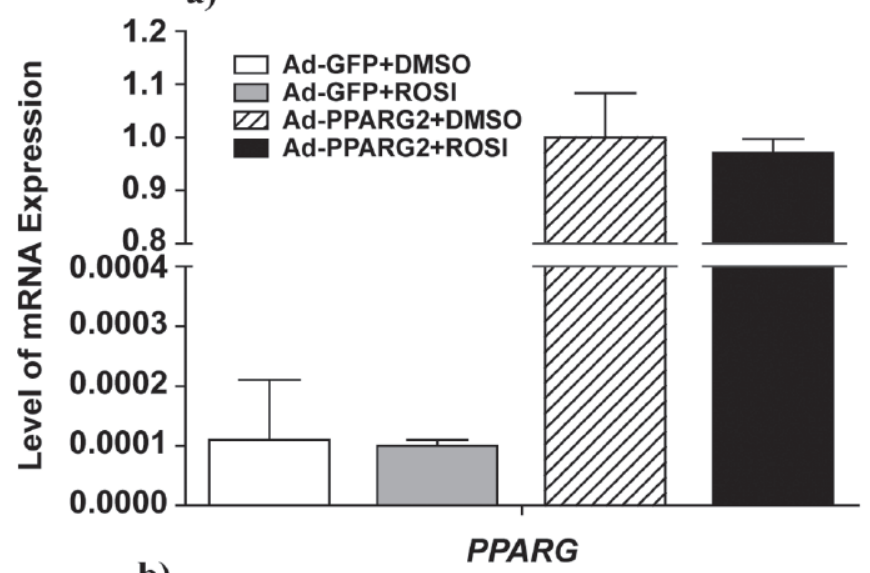

b)

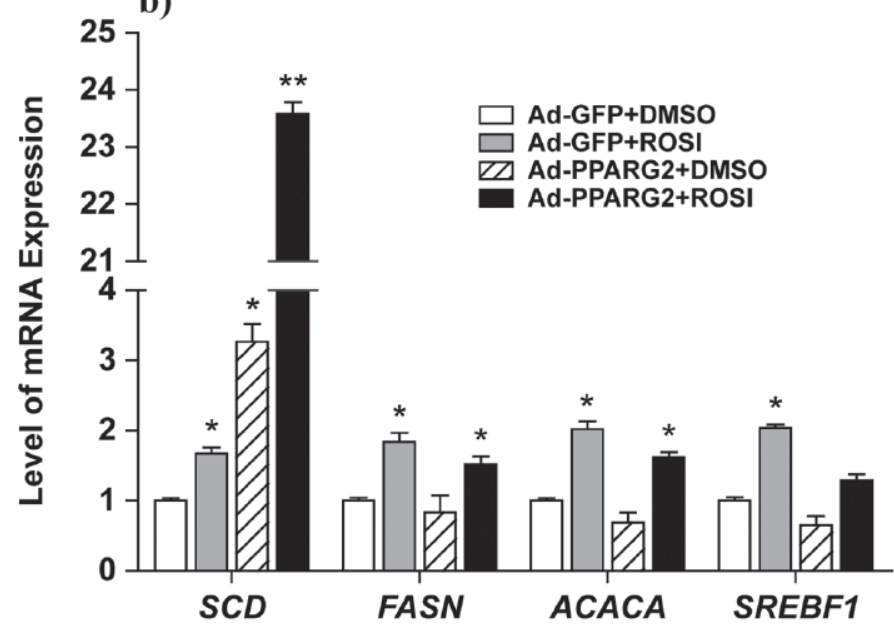

c) d)

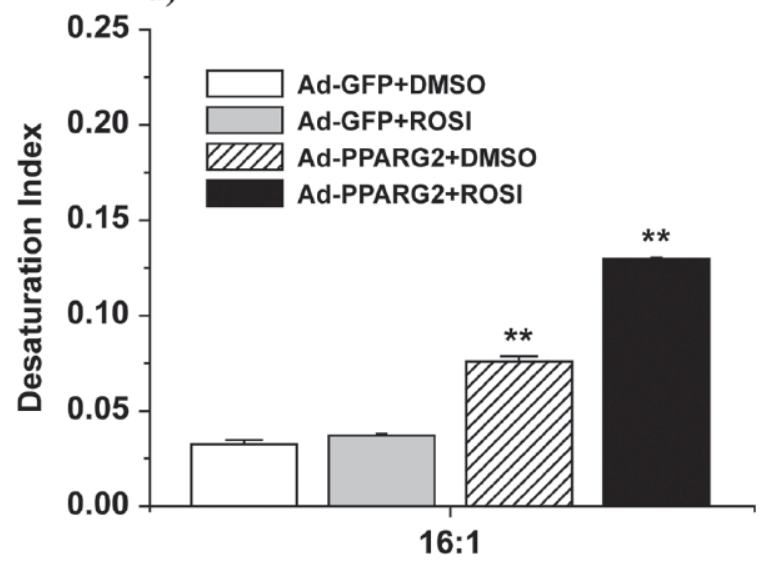

e)

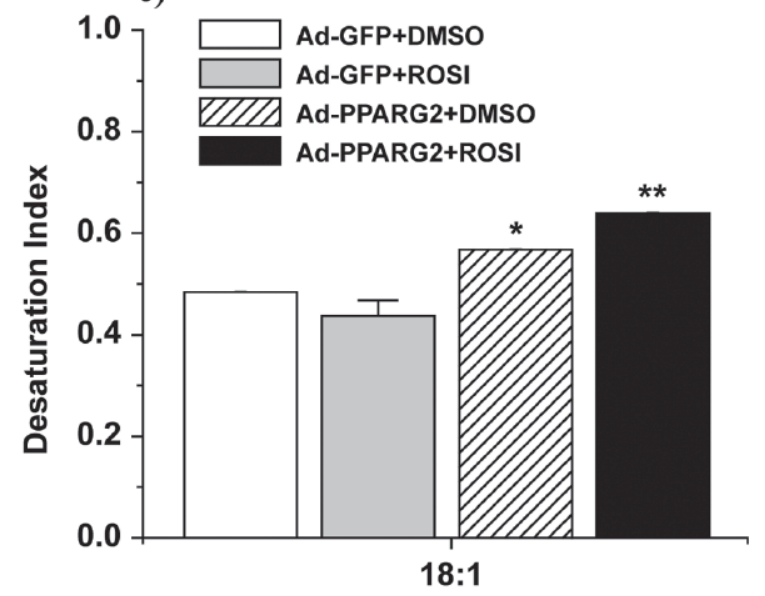

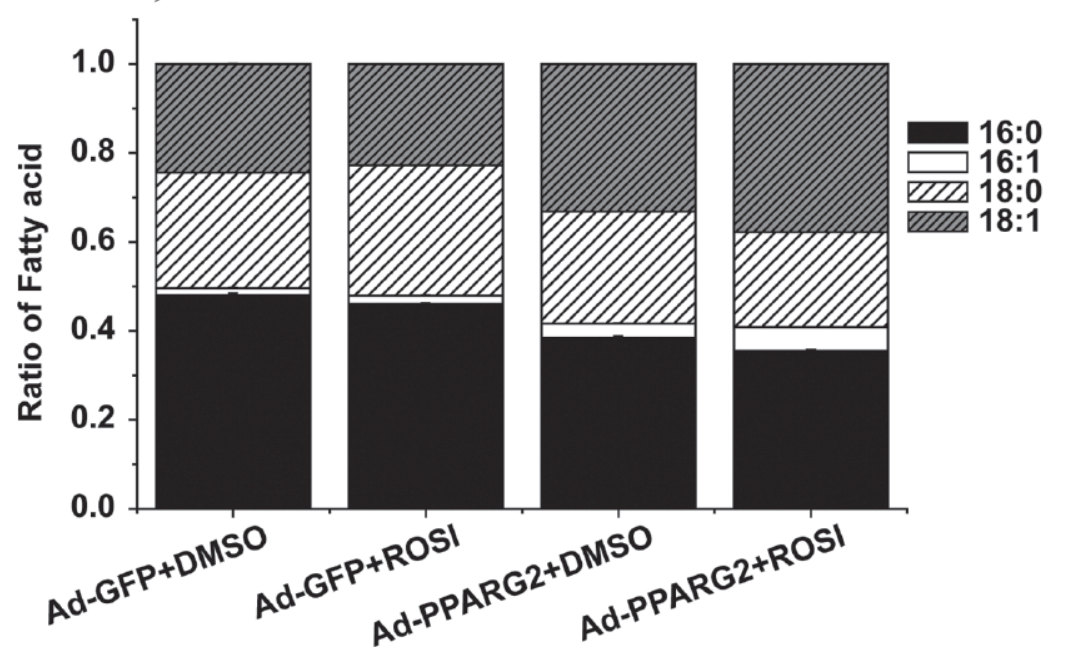

Figure 4. Effect of overexpression of peroxisome proliferator-activated receptor- $\gamma 2$ (PPARG2) on gene expression and FA concentration. After being infected with adenovirus Ad-PPARG2 or adenovirus containing green fluorescent protein (Ad-GFP) for 24 h, the goat mammary epithelial cells (GMEC) were cultured with rosiglitazone (ROSI) or dimethyl sulfoxide (DMSO) for $24 \mathrm{~h}$ and harvested for RNA and lipid extraction. (a) Level of PPARG expression after overexpression of PPARG2. (b) Level of FA synthase (FASN), acetyl-CoA carboxylase $\alpha$ (ACACA), stearoyl-CoA desaturase $(S C D)$, and sterol regulatory element-binding factor 1 (SREBF1) expression after overexpression of PPARG2. (c) Composition of 16- to 18-carbon FA in cells. Data are reported as ratios of total FA. (d) Desaturation index of 16:1. (e) Desaturation index of 18:1. Values are means \pm SEM for 3 individual experiments. Data of quantitative PCR were normalized to GAPDH and expressed relative to the Ad-GFP+DMSO (control) treatment. Data of desaturation indices are expressed relative to the Ad-GFP+DMSO (control) group. ${ }^{*} P<0.05$ and ${ }^{* *} P<0.01$ compared with the Ad-GFP+DMSO (control) group. 
gests that this isoform may play a role as a competitive inhibitor of PPARG1 for binding to the PPAR-response element site in GMEC. It also is possible that this isoform serves to exert some control on the lipogenic process by preventing excessive synthesis of mRNA of lipogenic genes (i.e., a counterregulatory mechanism over PPARG1).

The pattern of increase and decrease in expression of $A C A C A$ and FASN agreed with that of SREBF1, which is consistent with SREBF1 directly regulating the expression of both genes in rodents (Horton et al., 2002). These results strongly indicate that PPARG directly regulates the expression of $S C D$, whereas the effect on expression of $F A S N$ and $A C A C A$ is mediated potentially via PPARG activation of SREBF1 (Bionaz et al., 2013).

In nonruminants, many tissues express PPARG1, whereas PPARG2 is adipose depot specific, where it is expressed at very high levels relative to other tissues (Tontonoz et al., 1994a). Previous reports have shown that PPARG2 but not PPARG1 can reactivate adipogenesis (Ren et al., 2002). In adipose cells, the PPARG2 A/B-domain in the $\mathrm{N}$ terminus plays a genespecific role in transactivation and cofactor recruitment (Bugge et al., 2009). Some evidence exists demonstrating that high levels of PPARG1 in the mouse liver are sufficient to induce the adipogenic transformation of hepatocytes (Yu et al., 2003).

In the present study, both overexpression of PPARG1 and PPARG2 in GMEC increased the expression of $S C D$ and the stimulation of $S C D$ expression was additive after the treatment with ROSI, leading to an increase of more than 60 fold over the control values (Figure $3 \mathrm{~B})$. These results agree with the role of PPARG in controlling expression of SCD in nonruminant adipose (Risérus et al., 2005), liver (Yu et al., 2003), and fibroblast cells (Tontonoz et al., 1994a,b). Additionally, the expression of $S C D$ also decreases significantly after the knockdown of PPARG in GMEC (Shi et al., 2013). Taken together, our data strongly suggest that PPARG may directly regulate $S C D$ expression in GMEC.

Some studies have reported that after being activated by ligands, PPARG increases the desaturation index in adipose tissue (Risérus et al., 2005; Yao-Borengasser et al., 2008). However, the PPARG isoform responsible and the exact mechanisms for this effect have not been fully resolved. Clearly, in the context of lactation, establishing if and how PPARG can alter the composition of MUFA through regulating SCD is important, namely because mammary PPARG is responsive to LCFA (Kadegowda et al., 2009) and its expression increases during lactation (Bionaz and Loor, 2008).

The fact that overexpression of both isoforms of PPARG led to marked upregulation of $S C D$ and in- creased the desaturation indices of $16: 1$ and $18: 1$ is consistent with the function of SCD protein, which is to catalyze the conversion of $16: 0$ and 18:0 to $16: 1$ and 18:1 (Miyazaki and Ntambi, 2003). Our data, however, are inconsistent with previous data from nonruminant adipose tissue, which suggested that the activation of PPARG by ROSI could only increase the desaturation index of 16:1 (Risérus et al., 2005). However, despite the marked upregulation of $S C D$ expression when PPARG was overexpressed, a relatively modest change was observed in cellular FA composition. Such discrepancy in responses could be partly explained by the lack of exogenous 16:0 and 18:0 availability for the enzymatic reaction, lack of an increase in SCD protein, and also posttranslational effects (Waters and Ntambi, 1996; Jones et al., 1998). The lack of a goat-specific antibody for SCD clearly is a limiting factor in the present study. Although such data would have been valuable, the fact remains that the control of SCD and MUFA (at least in nonruminants) is primarily a result of changes in mRNA expression (Miyazaki and Ntambi, 2003).

Besides the control of MUFA synthesis at the level of SCD, other factors may exist that in coordination with the desaturation process affect the cellular FA composition [e.g., 1-acylglycerol-3-phosphate O-acyltransferase 6 (AGPAT6) or diacylglycerol acyltransferase 1 (DGAT1) mRNA and protein expression]. Both DGAT1 and AGPAT6 are integral components of the overall pathway leading to synthesis of milk lipid droplets and have a major effect on milk fat yield and composition (Thaller et al., 2003; Schennink et al., 2007; Demeter et al., 2009). The expression of DGAT1 and AGPAT6 in MacT cells increased significantly due to ROSI (Kadegowda et al., 2009). Some evidence also exists suggesting that these genes might be targets of PPARG in ruminant mammary cells (Bionaz et al., 2013).

In summary, the mechanism leading to an increase in the desaturation indices of $16: 1$ and $18: 1$ by PPARG1 and $P P A R G 2$ overexpression likely involves an increase in $S C D$ mRNA expression and possibly activity. Our data highlight the role of PPARG in GMEC in terms of regulating the desaturation of 16:0 and 18:0 and suggest that this nuclear receptor might be an important target for regulating the composition of MUFA in milk by nutritional means. Further studies are necessary to clarify these aspects of regulation.

\section{CONCLUSIONS}

The combination of ligand activation and gene isoform overexpression suggested that PPARG regulates the expression of $S C D$ and the composition of MUFA in GMEC. The response to overexpression of PPARG2 suggests that this isoform may play a counterregu- 
latory mechanism as a way to control de novo lipid synthesis. More controlled molecular experiments, such as promoter analysis and protein expression of target enzymes, will have to be performed in the future to address questions about the precise mechanisms of PPARG regulation of $S C D$. Together, results from the present study provide strong evidence that PPARG is the key regulator of MUFA and de novo FA synthesis (potentially via SREBF1). Understanding the regulatory role of PPARG in lipid metabolism in the lactating ruminant may contribute to improving the efficiency and quality of milk production.

\section{ACKNOWLEDGMENTS}

This research was jointly supported by the National Natural Science Foundation of China (Beijing, China; 31072013) and the Special Fund for Agro-Scientific Research in the Public Interest (China; 201103038). The authors thank James Hudson and Simon Foster from the University of Queensland (Brisbane, Australia) for initial input on the manuscript.

\section{REFERENCES}

Bauman, D. E., D. L. Ingle, R. W. Mellenberger, and C. L. Davis. 1973. Factors affecting in vitro lipogenesis by bovine mammary tissue slices. J. Dairy Sci. 56:1520-1525.

Bernard, L., J. Rouel, C. Leroux, A. Ferlay, Y. Faulconnier, P. Legrand, and Y. Chilliard. 2005. Mammary lipid metabolism and milk fatty acid secretion in alpine goats fed vegetable lipids. J. Dairy Sci. 88:1478-1489.

Bionaz, M., S. Chen, M. J. Khan, and J. J. Loor. 2013. Functional role of PPARs in ruminants: Potential targets for fine-tuning metabolism during growth and lactation. PPAR Res. 2013:684159.

Bionaz, M., and J. J. Loor. 2008. Gene networks driving bovine milk fat synthesis during the lactation cycle. BMC Genomics 9:366.

Bugge, A., L. Grøntved, M. M. Aagaard, R. Borup, and S. Mandrup. 2009. The PPAR $\gamma 2$ A/B-domain plays a gene-specific role in transactivation and cofactor recruitment. Mol. Endocrinol. 23:794-808.

Conte, G., M. Mele, S. Chessa, B. Castiglioni, A. Serra, G. Pagnacco, and P. Secchiari. 2010. Diacylglycerol acyltransferase 1, stearoylCoA desaturase 1, and sterol regulatory element binding protein 1 gene polymorphisms and milk fatty acid composition in Italian Brown cattle. J. Dairy Sci. 93:753-763.

Demeter, R. M., G. C. B. Schopen, A. G. J. M. Oude Lansink, M. P M. Meuwissen, and J. A. M. Arendonk. 2009. Effects of milk fat composition, DGAT1, and SCD1 on fertility traits in Dutch Holstein cattle. J. Dairy Sci. 92:5720-5729.

Elbrecht, A., Y. Chen, C. A. Cullinan, N. Hayes, M. Leibowitz, D. E. Moller, and J. Berger. 1996. Molecular cloning, expression and characterization of human peroxisome proliferator activated receptors gamma 1 and gamma 2. Biochem. Biophys. Res. Commun. 224:431-437.

Gou, D., T. Weng, Y. Wang, Z. Wang, H. Zhang, L. Gao, Z. Chen, P. Wang, and L. Liu. 2007. A novel approach for the construction of multiple shRNA expression vectors. J. Gene Med. 9:751-763.

Haenlein, G. F. W. 2004. Goat milk in human nutrition. Small Rumin. Res. 51:155-163.

Horton, J. D., J. L. Goldstein, and M. S. Brown. 2002. SREBPs: Activators of the complete program of cholesterol and fatty acid synthesis in the liver. J. Clin. Invest. 109:1125-1131.
Jacobs, A. A. A., J. Dijkstra, W. H. Hendriks, J. van Baal, and A. M. van Vuuren. 2013. Comparison between stearoyl-CoA desaturase expression in milk somatic cells and in mammary tissue of lactating dairy cows. J. Anim. Physiol. Anim. Nutr. (Berl.) 97:353-362.

Jones, B. H., M. K. Standridge, K. J. Claycombe, P. J. Smith, and N. Moustaïd-Moussa. 1998. Glucose induces expression of stearoylCoA desaturase in 3T3-L1 adipocytes. Biochem. J. 335:405-408.

Kadegowda, A. K. G., M. Bionaz, L. S. Piperova, R. A. Erdman, and J. J. Loor. 2009. Peroxisome proliferator-activated receptor- $\gamma$ activation and long-chain fatty acids alter lipogenic gene networks in bovine mammary epithelial cells to various extents. J. Dairy Sci. $92: 4276-4289$

Kgwatalala, P. M., E. M. Ibeagha-Awemu, A. F. Mustafa, and X. Zhao. 2009. Stearoyl-CoA desaturase 1 genotype and stage of lactation influences milk fatty acid composition of Canadian Holstein cows. Anim. Genet. 40:609-615.

Kolak, M., H. Yki-Järvinen, K. Kannisto, M. Tiikkainen, A. Hamsten, P. Eriksson, and R. M. Fisher. 2007. Effects of chronic rosiglitazone therapy on gene expression in human adipose tissue in vivo in patients with type 2 diabetes. J. Clin. Endocrinol. Metab. 92:720-724

Lin, X., J. Luo, L. Zhang, W. Wang, H. Shi, and J. Zhu. 2013. miR27a suppresses triglyceride accumulation and affects gene mRNA expression associated with fat metabolism in dairy goat mammary gland epithelial cells. Gene 521:15-23.

Loor, J. J., and J. H. Herbein. 2003. Reduced fatty acid synthesis and desaturation due to exogenous trans10,cis12-CLA in cows fed oleic or linoleic Oil. J. Dairy Sci. 86:1354-1369.

Luo, J., Z.-L. Deng, X. Luo, N. Tang, W.-X. Song, J. Chen, K. A Sharff, H. H. Luu, R. C. Haydon, K. W. Kinzler, B. Vogelstein, and T.-C. He. 2007. A protocol for rapid generation of recombinant adenoviruses using the AdEasy system. Nat. Protoc. 2:1236-1247.

Mele, M., G. Conte, B. Castiglioni, S. Chessa, N. P. P. Macciotta, A Serra, A. Buccioni, G. Pagnacco, and P. Secchiari. 2007. Stearoylcoenzyme A desaturase gene polymorphism and milk fatty acid composition in Italian Holsteins. J. Dairy Sci. 90:4458-4465.

Mele, M., R. Dal Zotto, M. Cassandro, G. Conte, A. Serra, A. Buccioni, G. Bittante, and P. Secchiari. 2009. Genetic parameters for conjugated linoleic acid, selected milk fatty acids, and milk fatty acid unsaturation of Italian Holstein-Friesian cows. J. Dairy Sci. 92:392-400.

Miyazaki, M., and J. M. Ntambi. 2003. Role of stearoyl-coenzyme A desaturase in lipid metabolism. Prostaglandins Leukot. Essent. Fatty Acids 68:113-121

Moioli, B., G. Contarini, A. Avalli, G. Catillo, L. Orrù, G. De Matteis, G. Masoero, and F. Napolitano. 2007. Short communication: Effect of stearoyl-coenzyme A desaturase polymorphism on fatty acid composition of milk. J. Dairy Sci. 90:3553-3558.

Ntambi, J. M. 1995. The regulation of stearoyl-COA desaturase (SCD). Prog. Lipid Res. 34:139-150.

Ostapchuk, P., and P. Hearing. 2005. Control of adenovirus packaging. J. Cell. Biochem. 96:25-35.

Peterson, D. G., E. A. Matitashvili, and D. E. Bauman. 2004. The inhibitory effect of trans-10, cis-12 CLA on lipid synthesis in bovine mammary epithelial cells involves reduced proteolytic activation of the transcription factor SREBP-1. J. Nutr. 134:2523-2527.

Pieniak-Lendzion, K., and R. Niedziółka. 2004. The importance of goat milk in human nutrition. Wiadomości Zootechniczne 42:39-44.

Reh, W. A., E. A. Maga, N. M. B. Collette, A. Moyer, J. S. ConradBrink, S. J. Taylor, E. J. DePeters, S. Oppenheim, J. D. Rowe, R H. BonDurant, G. B. Anderson, and J. D. Murray. 2004. Hot topic: Using a stearoyl-CoA desaturase transgene to alter milk fatty acid composition. J. Dairy Sci. 87:3510-3514.

Ren, D., T. N. Collingwood, E. J. Rebar, A. P. Wolffe, and H. S. Camp. 2002. PPAR $\gamma$ knockdown by engineered transcription factors: Exogenous PPAR $\gamma 2$ but not PPAR $\gamma 1$ reactivates adipogenesis. Genes Dev. 16:27-32

Risérus, U., G. D. Tan, B. A. Fielding, M. J. Neville, J. Currie, D. B. Savage, V. K. Chatterjee, K. N. Frayn, S. O'Rahilly, and F. Karpe. 2005. Rosiglitazone increases indexes of stearoyl-CoA desaturase activity in humans: Link to insulin sensitization and the role of 
dominant-negative mutation in peroxisome proliferator-activated receptor- $\gamma$. Diabetes 54:1379-1384.

Rosen, E. D., and B. M. Spiegelman. 2001. PPARgamma: A nuclear regulator of metabolism, differentiation, and cell growth. J. Biol. Chem. 276:37731-37734.

Schennink, A., W. M. Stoop, M. H. P. W. Visker, J. M. L. Heck, H. Bovenhuis, J. J. van der Poel, H. J. F. van Valenberg, and J. A. M. van Arendonk. 2007. DGAT1 underlies large genetic variation in milk-fat composition of dairy cows. Anim. Genet. 38:467-473.

Shi, H., J. Luo, Y. Zhu, Z. Wang, and W. Zhao. 2012. Cloning, sequence analysis and over-expression of $S C D$ gene of dairy goat. China Agric. Sci. 45:5091-5101.

Shi, H., J. Luo, J. Zhu, J. Li, Y. Sun, X. Lin, L. Zhang, D. Yao, and H. Shi. 2013. PPAR $\gamma$ regulates genes involved in triacylglycerol synthesis and secretion in mammary gland epithelial cells of dairy goats. PPAR Res. 2013:310948.

Shimano, H. 2001. Sterol regulatory element-binding proteins (SREBPs): Transcriptional regulators of lipid synthetic genes. Prog. Lipid Res. 40:439-452.

Singh Ahuja, H., S. Liu, D. L. Crombie, M. Boehm, M. D. Leibowitz, R. A. Heyman, C. Depre, L. Nagy, P. Tontonoz, and P. J. A. Davies. 2001. Differential effects of rexinoids and thiazolidinediones on metabolic gene expression in diabetic rodents. Mol. Pharmacol. 59:765-773.

Thaller, G., W. Kramer, A. Winter, B. Kaupe, G. Erhardt, and R. Fries. 2003. Effects of DGAT1 variants on milk production traits in German cattle breeds. J. Anim. Sci. 81:1911.

Tontonoz, P., E. Hu, R. A. Graves, A. I. Budavari, and B. M. Spiegelman. 1994a. mPPAR gamma 2: Tissue-specific regulator of an adipocyte enhancer. Genes Dev. 8:1224-1234.

Tontonoz, P., E. Hu, and B. M. Spiegelman. 1994b. Stimulation of adipogenesis in fibroblasts by PPAR $\gamma 2$, a lipid-activated transcription factor. Cell 79:1147-1156.
Walkey, C. J., and B. M. Spiegelman. 2008. A functional peroxisome proliferator-activated receptor- $\gamma$ ligand-binding domain is not required for adipogenesis. J. Biol. Chem. 283:24290-24294.

Wang, W., J. Luo, Y. Zhong, X. Lin, H. Shi, J. Zhu, J. Li, Y. Sun, and W. Zhao. 2012. Goat liver X receptor $\alpha$, molecular cloning, functional characterization and regulating fatty acid synthesis in epithelial cells of goat mammary glands. Gene 505:114-120.

Wang, Z., J. Luo, W. Wang, W. Zhao, and X. Lin. 2010. Characterization and culture of isolated primary dairy goat mammary gland epithelial cells. Sheng Wu Gong Cheng Xue Bao 26:1123-1127.

Waters, K. M., and J. M. Ntambi. 1996. Polyunsaturated fatty acids inhibit hepatic stearoyl-CoA desaturase-1 gene in diabetic mice. Lipids 31:S33-S36.

Way, J. M., W. W. Harrington, K. K. Brown, W. K. Gottschalk, S. S. Sundseth, T. A. Mansfield, R. K. Ramachandran, T. M. Willson, and S. A. Kliewer. 2001. Comprehensive messenger ribonucleic acid profiling reveals that peroxisome proliferator-activated receptor $\gamma$ activation has coordinate effects on gene expression in multiple insulin-sensitive tissues. Endocrinology 142:1269-1277.

Yao-Borengasser, A., N. Rassouli, V. Varma, A. M. Bodles, N. Rasouli, R. Unal, B. Phanavanh, G. Ranganathan, R. E. McGehee Jr., and P. A. Kern. 2008. Stearoyl-coenzyme A desaturase 1 gene expression increases after pioglitazone treatment and is associated with peroxisomal proliferator-activated receptor- $\gamma$ responsiveness. J. Clin. Endocrinol. Metab. 93:4431-4439.

Yu, S., K. Matsusue, P. Kashireddy, W.-Q. Cao, V. Yeldandi, A. V. Yeldandi, M. S. Rao, F. J. Gonzalez, and J. K. Reddy. 2003. Adipocyte-specific gene expression and adipogenic steatosis in the mouse liver due to peroxisome proliferator-activated receptor $\gamma 1$ (PPAR $\gamma 1)$ overexpression. J. Biol. Chem. 278:498-505. 